\title{
ON AND OFF SUBLAMINAE IN THE LATERAL GENICULATE NUCLEUS OF THE FERRET ${ }^{1}$
}

\author{
MICHAEL P. STRYKER ${ }^{2}$ AND KATHLEEN R. ZAHS \\ Department of Physiology and Division of Neuroscience, University of California, San Francisco, California 94143
}

Received November 2, 1982; Revised March 7, 1983; Accepted March 28, 1983

\begin{abstract}
Like the retinal ganglion cells from which they receive their input, most relay neurons in the lateral geniculate nucleus have $\mathrm{ON}$ - or $\mathrm{OFF}$-center receptive fields with antagonistic surrounds. In the cat, neurons with these two types of receptive fields are anatomically intermingled, even though the $\mathrm{ON}$ and $\mathrm{OFF}$ systems are functionally segregated. In the ferret, there is a sublamination of the retinal input to lateral geniculate nucleus laminae A and A1. We have investigated the function of this sublamination by making microelectrode recordings and have found that each sublamina consists of geniculate neurons of a single center type.
\end{abstract}

In higher mammals, information about lightness and darkness in the retinal image appears to be conveyed from the eye to the brain in parallel channels of $\mathrm{ON}$ and OFF-center retinal ganglion cell axons (Kuffler, 1953). In the retina, segregation of center types is accomplished by the arborization of depolarizing bipolar cells to contact ON-center ganglion cells within a different sublamina of the inner plexiform layer from that in which hyperpolarizing bipolar cells contact the OFF-center cells (Famiglietti et al., 1976). These ON and OFF channels remain at least largely separate through the synapses of the lateral geniculate nucleus (LGN) (Hubel and Wiesel, 1961; Cleland et al., 1971).

Reports from Schiller's laboratory of a strict laminar segregation of center type in the LGN of the tree shrew (Conway et al., 1980) led us to wonder whether the subtle bisublamination of the main (A) laminae of the ferret LGN, noted by Sanderson (1974) and Guillery and coworkers (Linden et al., 1981) and termed "leaflets," might provide a substratc for the maintenance of separate $\mathrm{ON}$ and OFF channels. Microelectrode recordings now reveal that each leaflet consists of geniculate cells of a single center type.

${ }^{1}$ This work was supported by Grants RO1-EY-02874 and KO4-EY-00213 from the National Institutes of Health, by research fellowships from the Sloan Foundation and the $\mathrm{Na}$ tional Foundation-March of Dimes, and by a predoctoral fellowship from the National Science Foundation. We wish to thank Dr. Simon LeVay for comments on an earlier version of this manuscript and Ms. Sheri Strickland for technical assistance.

${ }^{2}$ To whom correspondence should be addressed, at Department of Physiology, S-762, University of California, San Francisco, CA 94143.

\section{Materials and Methods}

Animals. Eight adult ferrets (Mustela putorius furo) were obtained from Marshall Research Animals, Inc., North Rose, NY, and housed in the University of California vivarium for 1 to 8 weeks. For all but two experiments, animals were females weighing between 700 and $850 \mathrm{gm}$; the others were 1.1 - to $1.2-\mathrm{kg}$ males.

Labeling retinal afferents. In three ferrets, retinal afferents to the LGN were labeled autoradiographically by intravitreal injections of 200 to $2000 \mu \mathrm{Ci}$ of $\left[{ }^{3} \mathrm{H}\right]$ proline (Amersham TRK.439, specific activity $40 \mathrm{Ci} / \mathrm{mmol}$ ). In these cases, an unlabeled or extremely lightly labeled gap was clearly seen between two tiers of label in both laminae A and A1. Following Sanderson (1974), we took the gap in the labeled afferents to indicate the dividing line between the sublaminae or "leaflets." Figure 1 shows such a labeled section, cut coronally from the side contralateral to the injected eye.

Inspecting such labeled sections along with neighboring sections stained by a Nissl method eventually allowed us to discern the locations of the leaflets in most stained sections without reference to the labeled ones. Although the ferret's geniculate nucleus lacks a cell-poor interleaflet plexus, knowledge of the form taken by the leaflets in labeled sections at each level guided us in picking out the subtle differences in neuronal architecture which mark the boundaries of the leaflets. A comparison of the labeled section shown in Figure 1 with the Nissl-stained section from another animal shown in Figure $2 A$ will make clear that it was possible to do this. On the most caudal coronal sections and the most lateral parasagittal sections, determining the borders of the leaflets on unlabeled sections by reference to our atlas series of labeled sections was sometimes difficult; rostrally and medially 
near the border with the medial intralaminar nucleus, it was sometimes impossible.

Retinal afferents in two other ferrets were labeled by injecting the vitreous humor of one eye with $0.25 \mathrm{mg}$ of wheat germ agglutinin conjugated to horseradish peroxidase (Sigma, catalogue no. L9008) 3 to 5 days before perfusion, and reacting brain sections with tetramethyl benzidine according to the protocol of Meslaum (1978). Figure 3 shows examples of sagittal sections labeled using this procedure.

Microelectrode recording. Eight ferrets were prepared for physiological recording using techniques which are conventional for studies of the cat visual system (Shatz and Stryker, 1978). Briefly, the ferret was initially anesthetized with a mixture of acepromazine and ketamine ( 0.04 and $40 \mathrm{mg} / \mathrm{kg}$, i.m., respectively) to allow cannulation of the femoral vein and trachea. Thiopentol sodium $(20 \mathrm{mg} / \mathrm{kg}$, i.v.) was employed to continue anesthesia throughout the rest of the surgery. The ferret was placed on a heating pad which kept body temperature at $38^{\circ} \mathrm{C}$ and fitted in a modified kitten stereotaxic instrument; then, the scalp, skull, and dura overlying the LGN were opened. Plastic contact lenses ( 2.7 to $2.9 \mathrm{~mm}$ base curve, plano) were fitted to focus the eyes on a tangent screen subtending $80^{\circ}$ at a distance of $57 \mathrm{~cm}$. This screen could be rotated to an angle of $60^{\circ}$ to the animal's midsagittal plane when necessary for plotting eccentric receptive fields. Focus, determined by the front surface power of the contact lens, was checked by retinoscopy, and the most appropriate lens was selected. The projections of the two optic discs were then plotted on the tangent screen using a reversing beam ophthalmoscope. Barbiturate infusion was discontinued and anesthesia was maintained by ventilating the ferret with $75 \%$ nitrous oxide, $25 \%$ oxygen at a rate and volume which maintained peak inspiratory pressure at $1.5 \mathrm{kPa}$ and end-tidal carbon dioxide at 3.8 to $4.3 \%$. Neuromuscular blockade was then induced using pancuronium bromide $(0.1 \mathrm{mg} /$ $\mathrm{kg}$-hr).

Lacquered tungsten microelectrodes (Hubel, 1957) were driven down to the LGN on a vertical axis in five ferrets or on an axis in the parasagittal plane inclined 40 to $45^{\circ}$ pointing anterior from the vertical in three ferrets. These electrodes had conical exposed tips tapering from 10 to $15 \mu \mathrm{m}$ diameter to a sharp point over a length of 20 to $30 \mu \mathrm{m}$. With impedances of 1 to $2 \mathrm{megohm}$ at 120 $\mathrm{Hz}$, they were designed to record multiunit activity, although they would sometimes isolate single units. Typically, 2 to 5 different spikes more than 4 times greater than the noise level $(50 \mu \mathrm{V})$ could be discerned. Such electrodes were advantageous for revealing clusters of units having similar response properties. At most sites within the LGN, all of the units recorded with such electrodes did have similar response properties (Zahs and Stryker, 1982; see Fig. $4, B$ and $C$, below): they were driven from the same eye and were of the same center type.

Assignment of recording sites to leaflets. One or more electrolytic lesions were made along the course of each electrode penetration by passing -4 to $-6 \mu \mathrm{A}$ for 4 to 6 sec. Nissl-stained sections containing the microelectrode penetrations were projected at a magnification of $\times 47$, and tracings, including all sublaminar boundaries, were made without knowledge of or reference to the physiological findings. It was then possible, by reference to microdrive readings at the microlesions and at the entry point into the LGN, to reconstruct the locations of all recording sites on these tracings. This procedure located the point of transition between responses from the two eyes accurately at the border between laminae A and Al. Only after assigning each recording site to the $\mathrm{C}$ laminae or to one of the leaflets of laminae A and A1 (or to the border zone between leaflets) were the physiological ON or OFF responses taken account of and noted on the tracings.

Two penetrations were made into caudal and medial regions of the LGN in which we were unable to identify the borders of the leaflets anatomically. Although ON and $\mathrm{OFF}$ responses were segregated here as they were elsewhere, data from the 28 recording sites along these penetrations are excluded from the present analysis.

Data from the initial three of the eight animals used in this study were not available for this correlation because the first two were used only to obtain stereotaxic coordinates and the third was sectioned in the wrong plane for reconstruction of the penetrations.

\section{Results}

Anatomy and physiology of LGNs cut in coronal section. The pattern of labeling of retinal afferents to the contralateral LGN is shown in Figure 1. This and subsequent coronal sections are shown with dorsal up and medial to the right. The two tiers of label in lamina $\mathrm{A}$ were found to be nearly continuous, and the overall pattern of labeling was similar to that described by Guillery (1971) and Linden et al. (1981). The principal difference between our results and theirs was that it was necessary for us to cut the brains coronally or parasagittally rather than horizontally, so that entire electrode tracks could be traced in one or a few sections.

A typical vertical penetration into the LGN is illustrated in Figure 2. When the electrode first passed into the nucleus, a region of high spontaneous activity and strong visual responses was invariably encountered. Histology later showed this region to be the $\mathrm{C}$ laminae of the LGN. The unit clusters here were frequently responsive to both eyes, depending on the eccentricity of the receptive fields in the visual field, and were usually responsive to both the onset and offset of a spot flashed in the center of the receptive field area. The single units isolated at these sites were always monocularly driven and of either ON- or OFF-center type, suggesting that in these laminae units of different types were intermingled within the resolving distance of the multi-unit microelectrode. Some unit clusters (38 of 138), however, were predominantly or exclusively of one sign of contrast, raising the possibility that a detailed organization may exist in these laminae beyond the resolution of our methods.

After a variable distance, the electrode next encountered a region of distinctly lower spontaneous activity and dramatically different response properties. Here, the unit clusters were driven exclusively by one eye and, in 225 of 245 cases, exclusively by either the onset or offset 


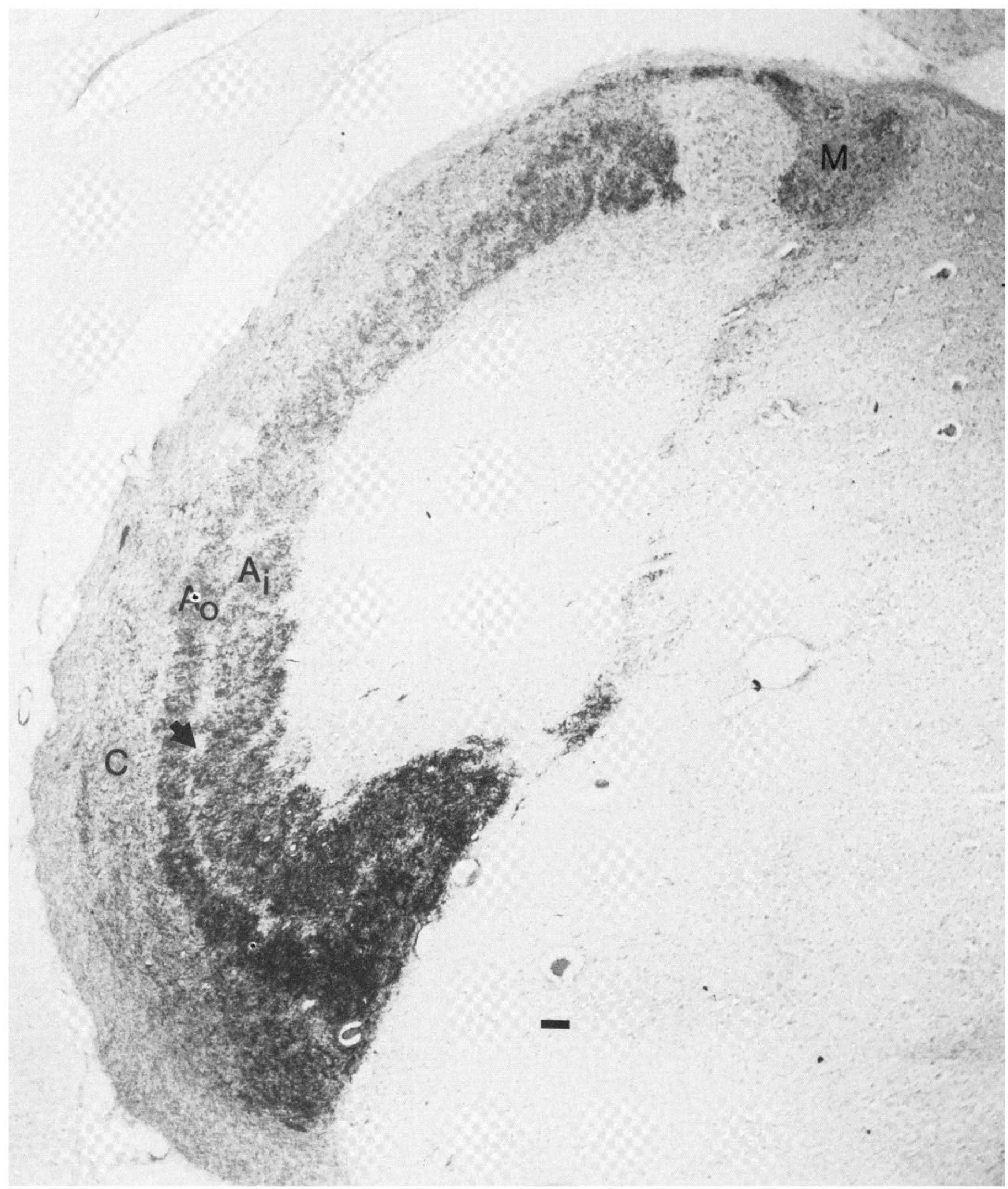

Figure 1. Autoradiograph shown in brightfield of a coronal section through a LGN contralateral to an eye in which the vitreous humor had been injected with $\left[{ }^{3} \mathrm{H}\right]$ proline. The section has been lightly stained with cresyl violet. Note two nearly continuous tiers of label in lamina A. $A_{o}$, outer leaflet; $A_{i}$, inner leaflet; $C, \mathrm{C}$ laminae; $M$, medial interlaminar nucleus. The arrowhead points to a lightly labeled gap between leaflets. Dorsal is up; medial is right. The scale bar is $100 \mu \mathrm{m}$. 

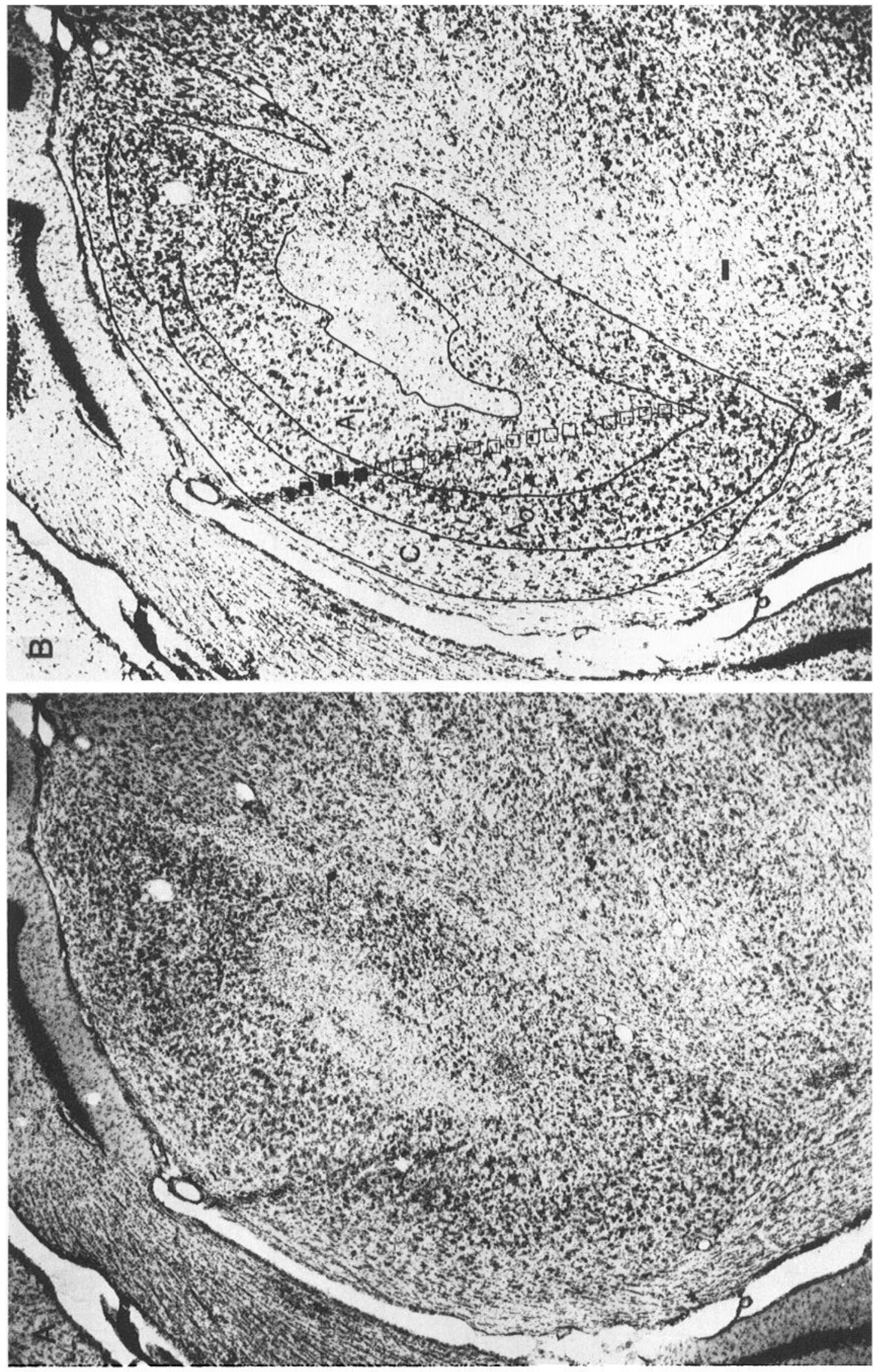

\& б요 \& 8 . 政 등 돈 을 क 중

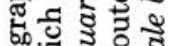
궁

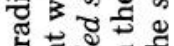
웅 卷. 동을 3 i 30 넝 융 농.

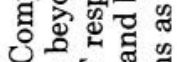
ठ०น क :

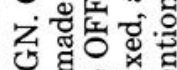
ज्व ฐ. 의. . ธ。

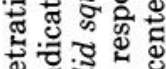
¿

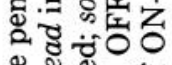

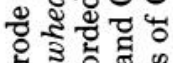
క 농정웡

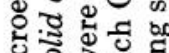
范 䗆 ฮై 过 $\triangleright . \Xi \pm$. ๘又晋 a 30 으 药 क्ष के

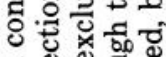
I

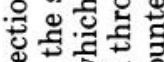
为菏

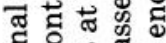
둔

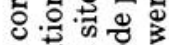
क 엉 뎡 ⿶凵 원

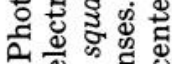
से है ง కั้

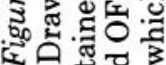
ผ 융 


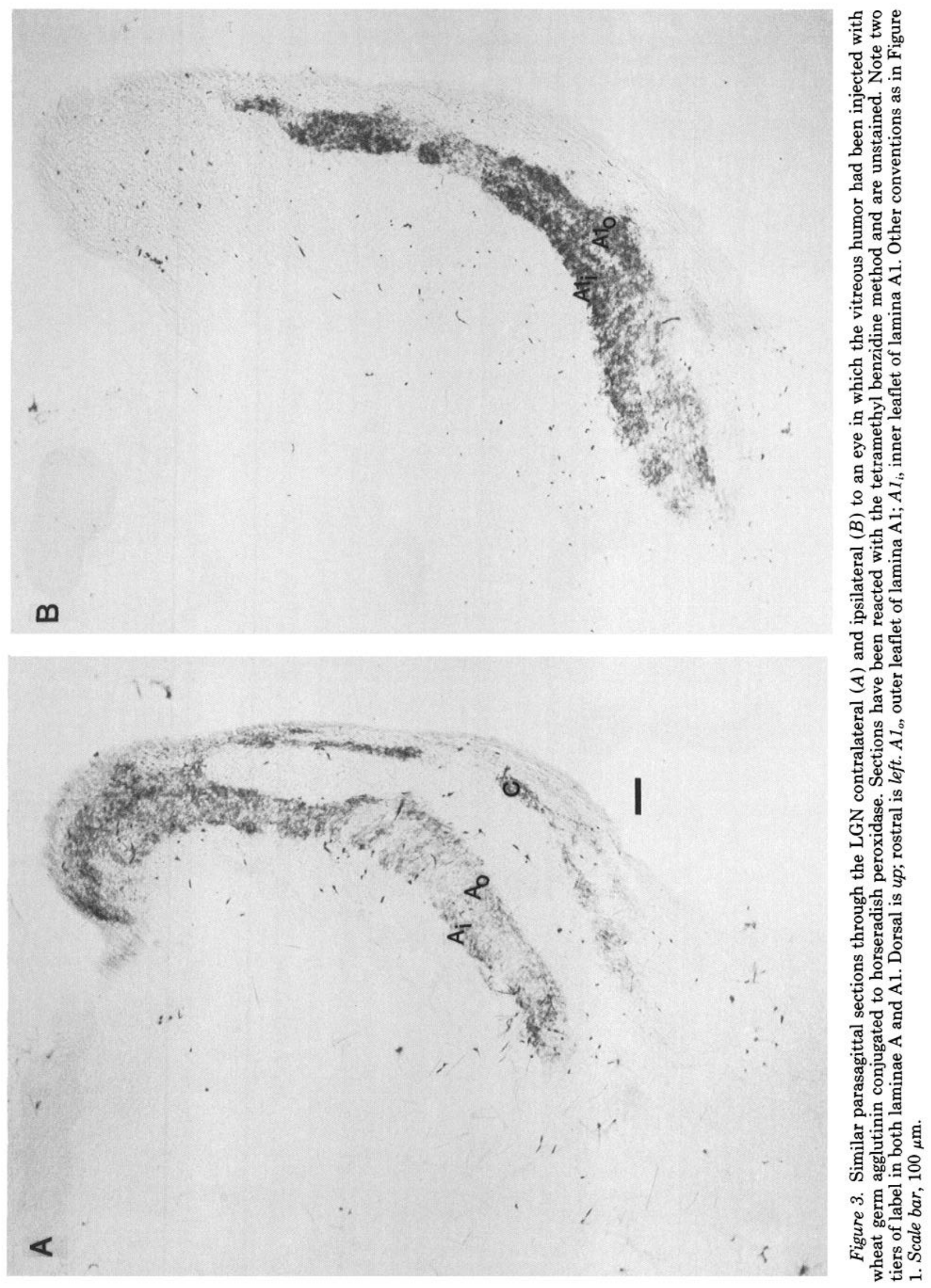


of illumination in the receptive field. All single units isolated at such sites shared these properties of the cluster and were found to have approximately circular receptive fields with mutually antagonistic center-surround organization similar to that found in the cat (Hubel and Wiesel, 1961).

We noted the eye and center type of unit clusters at $100-\mu \mathrm{m}$ intervals along each penetration. As the penetration continued downward and receptive fields progressed down the visual field (Zahs and Stryker, 1982), the eye and center type could change or could remain the same for distances greater than $1 \mathrm{~mm}$. The penetration shown in Figure 2, for example, passed briefly through a region of contralateral eye $\mathrm{OFF}$ responses before entering an
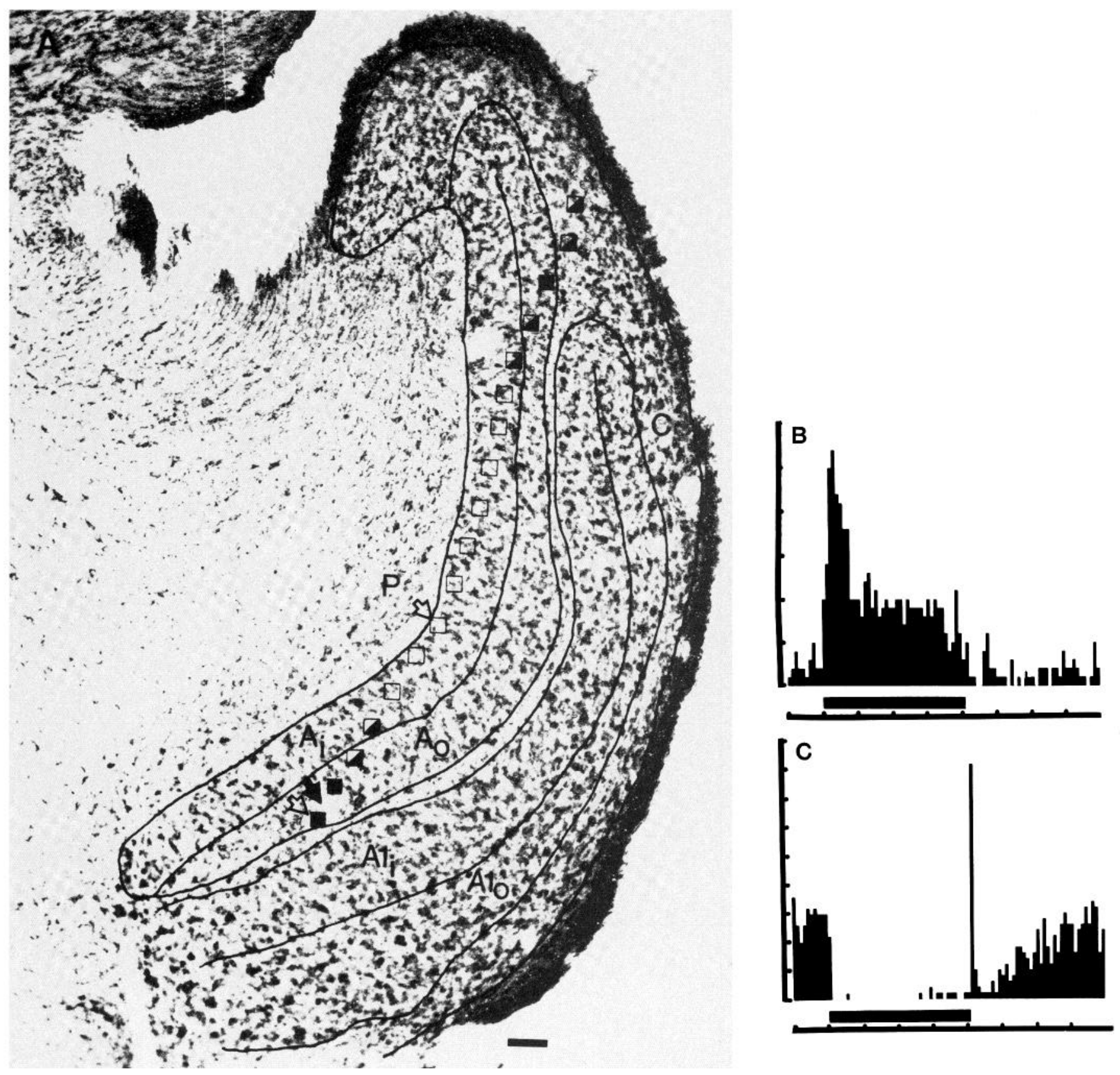

Figure 4. A, Vertical electrode penetration into the LGN drawn onto a photograph of a Nissl-stained parasagittal section. The upper open arrowhead indicates the site at which the poststimulus histogram in $B$ was obtained; the lower open arrowhead indicates the site of the poststimulus histogram in $C$. The solid arrowhead indicates the site of the electrolytic marking lesion. $P$, perigeniculate nucleus. Other conventions as in Figures 2 and 3 . The scale bar is $100 \mu \mathrm{m}$. B, Poststimulus histogram for the site indicated by the upper open arrowhead in $A$. This histogram was compiled from a multi-unit record consisting of at least four single units. Note that all gave only an $\mathrm{ON}$ response. Tic marks indicate $500 \mathrm{msec}$ and 10 spikes/sec. The heavy black line on the ordinate indicates the period in which the stimulus light was on. $C$, Poststimulus histogram for the site indicated by the lower open arrowhead in $A$. This histogram was compiled from multiple units, all of which responded only at OFF. Conventions as in B. 
extended region of $\mathrm{ON}$ responses. Receptive fields following the last one indicated on Figure 2 were not plotted because of their extreme inferior position in the visual field. Only in regions of transition between eyes or center types did the electrode record mixed activity, and single units were always monocular and either ON- or OFFcenter.

When such penetrations were located on histological sections by reference to electrolytic lesions made along their course, the $\mathrm{ON}$ and $\mathrm{OFF}$ regions were seen to correspond to the sublaminae or leaflets of layers A and A1 of the LGN.

Anatomy and physiology of LGNs cut in parasagittal section. The distribution of retinal afferents labeled by intraocular injection of wheat germ agglutinin-horseradish peroxidase is shown in parasagittal section in Figure 3. This and subsequent sections are shown with dorsal up and rostral to the left. Two tiers of label were found in each of laminae A and A1. The overall pattern of labeling was similar to that seen in the autoradiographically labeled sections except that fascicles of peroxidase reaction product were seen in the border zone between the leaflets.

In parasagittal sections labeled by either method, the dense labeling at the outsides of each leaflet was sometimes discontinuous in one or both tiers. This observation raises the possibility that the sublaminae are not completely continuous. We were unable to confirm this possibility with physiological recordings, for the electrode never passed through such a site in a labeled animal. It is possible that this occasional discontinuity is artifact due to incomplete labeling of or damage to the retinal ganglion cells or axons which would supply input to the unlabeled area.

Figure 4 shows a vertical electrode penetration in parasagittal section. This penetration passed through high spontaneous mixed $\mathrm{ON}$ and OFF activity in the $\mathrm{C}$ laminae and OFF responses in the outer leaflet of lamina A before encountering $\mathrm{ON}$ responses in the inner leaflet of lamina A. It remained within this leaflet until nearly the end of the penetration, when it re-entered the outer leaflet of lamina $A$, again encountering $\mathrm{OFF}$ responses. Figure $4, B$ and $C$, shows poststimulus histograms for unit clusters, each containing at least four units, at two sites along this penetration. Note that only an $\mathrm{ON}$ response is seen in Figure $4 B$, taken at the location in the inner leaflet indicated by the upper open arrow, and only an $\mathrm{OFF}$ response, taken at the location in the outer leaflet indicated by the lower open arrow, is seen in Figure $4 C$. Such histograms show responses typical of more than $90 \%$ of the multi-unit responses in the A laminae.

The sublaminar distribution of 383 recording sites in 41 penetrations in five ferrets is shown in Table I. Note that within the A laminae, mixed $\mathrm{ON}$ and OFF responses were encountered primarily at recording sites located at the borders of leaflets. Only $8 \%$ of recording sites had any contribution from responses of the opposite center type.

The arrangement of the sublaminae was made clearer by making penetrations as nearly normal to the geniculate laminae as could be managed without removing the tentorium. Examples of two such penetrations are shown
TABLE I

Visual response properties by sublamina

The distribution of LGN recording sites by sublamina and visual response properties. Sites characterized as "ON" were unanimously so: all audible units at those sites responded exclusively to the onset of a light spot of the appropriate size centered in the receptive field. At the sites classified as predominantly $\mathrm{ON}(\mathrm{ON} \gg \mathrm{OFF})$, nearly all the units responded to light onset, but a weak or distant response was also obtained to the offset of the receptive field spot. It was not always possible to determine whether this OFF response emanated from an OFF-center geniculate cell or was a surround response of a small $\mathrm{ON}$ center unit whose receptive field did not overlap those of the other simultaneously recorded units. The $\mathrm{OFF}$ and $\mathrm{OFF} \gg \mathrm{ON}$ categories were defined similarly. The "mixed" category includes all sites at which a substantial response was present at both ON and OFF; in most cases it was clear that such responses emanated from two separate populations of units. For the locations of the named sublaminae, refer to Figures $2 B$ or $3 B$. "Border zone" refers to sites on the border of two leaflets.

\begin{tabular}{lrcccrc}
\hline \multicolumn{1}{c}{$\begin{array}{c}\text { Location } \\
\text { Response }\end{array}$} & ON & ON $\gg$ OFF & Mixed & OFF $\gg$ ON & OFF & Total \\
\hline A outer & 1 & 0 & 0 & 2 & 53 & 56 \\
A inner & 95 & 2 & 0 & 0 & 0 & 97 \\
A1 outer & 1 & 0 & 0 & 0 & 24 & 25 \\
Al inner & 51 & 1 & 0 & 0 & 0 & 52 \\
Border zone & 0 & 0 & 13 & 2 & 0 & 15 \\
C & 7 & 2 & 100 & 21 & 8 & 138 \\
Total & 155 & 5 & 113 & 25 & 85 & 383 \\
\hline
\end{tabular}

in Figure 5. The more dorsal penetration nearly followed the lines of projection of the visual field so that receptive field position changed very little along its course. The sequence of response types in such penetrations was invariably as follows: first, C laminae, with high spontaneous activity and usually mixed responses; then, unless the receptive fields were in the monocular segment of the visual field, the outer leaflet of lamina A1 containing ipsilateral eye OFF-center units followed by the inner leaflet of $\mathrm{A} 1$ containing ipsilateral $\mathrm{ON}$-center units; and finally, the outer leaflet of layer A with contralateral OFF-center units followed by the inner leaflet with ONcenter units. The electrode usually then passed into the perigeniculate nucleus in which responses and receptive field positions were much different.

\section{Discussion}

The present findings indicate that $\mathrm{ON}$ - and OFFcenter geniculate cells are segregated from each other in the A laminae of the ferret's LGN: responses at only 6 recording sites of 230 within anatomical leaflets had any contribution of the inappropriate center type. They suggest strongly that the terminal arbors of retinal afferents are also segregated by center type; indeed, discovering the functional correlate of the stratification of the retinal afferents was the original goal of this study.

These findings shed little light on whether $\mathrm{ON}$ - and OFF-center cells are segregated in the $\mathrm{C}$ laminae. Our multi-unit electrodes found only 38 of 138 sites to be predominantly or exclusively of one center type, but such electrodes may sample over a distance that is considerable compared to the width of possible sublaminae in the C laminae.

Sublamination may be a general feature of organiza- 


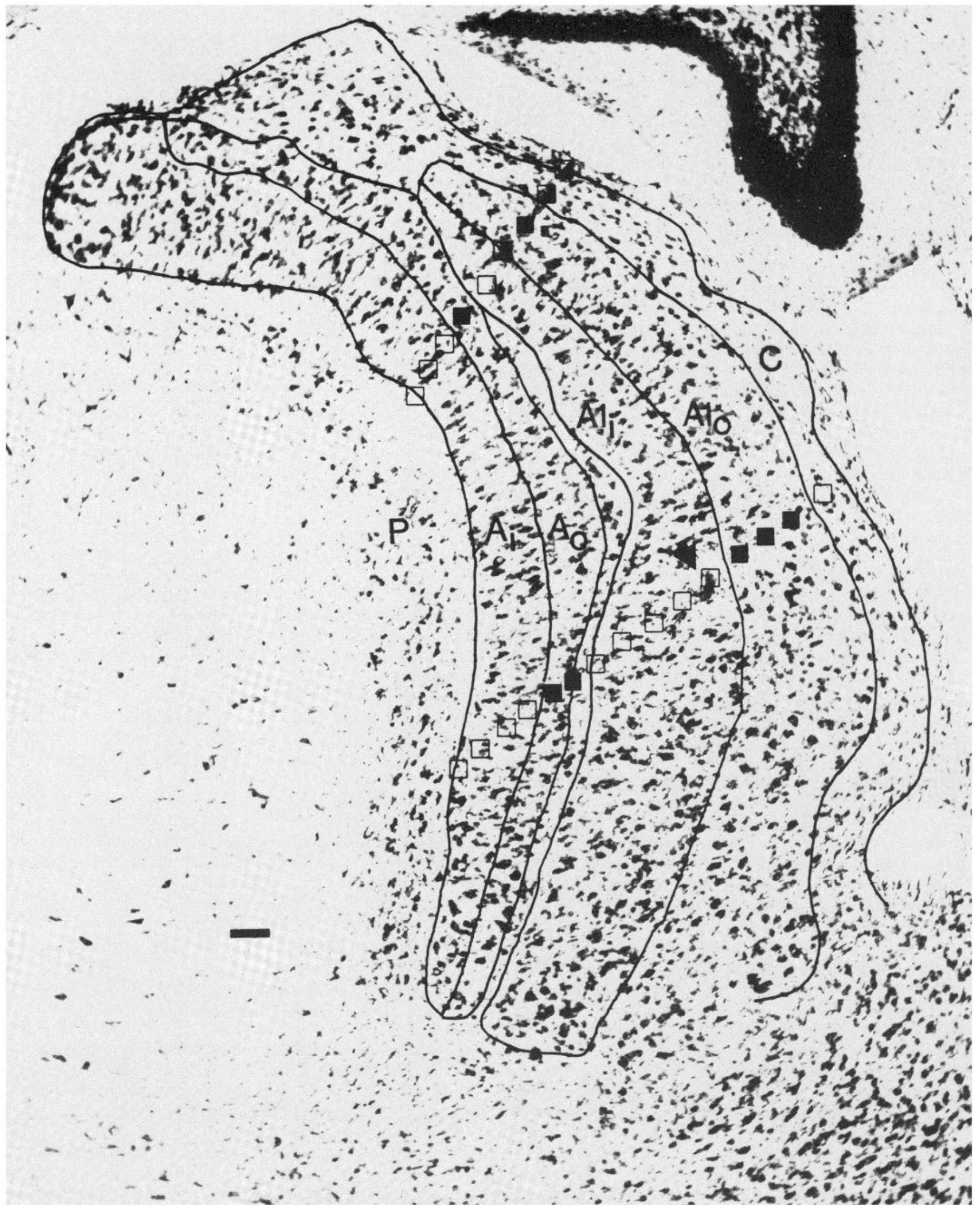

Figure 5. Two electrode penetrations inclined in the parasagittal plane drawn onto a photograph of a Nissl-stained parasagittal section through the LGN. Note the sequence of responses encountered as the electrode passed through the leaflets. The arrowhead indicates the site of the electrolytic marking lesion. Other conventions as in Figure 4. 
tion by which different response properties can remain segregated from one another through stages of synaptic relay. In the $L G N$, a strict lamination or sublamination of $\mathrm{ON}$ - and $\mathrm{OFF}$-center units like that in the ferret has been observed in the tree shrew (Conway et al., 1980) and mink (LeVay and McConnell, 1982). But sublamination may be covert; the retina was studied for many years before the function of $\mathrm{ON}$ and OFF sublamination in the inner plexiform layer was noted. If such sublamination were not strict, it would be difficult to discover with single-unit recording techniques. Perhaps a messy or incomplete sublamination may underly the intermingled response types found in the LGN of other animals, as results from the monkey suggest (Schiller and Malpeli, 1978).

Several questions remain about the sublamination observed in the ferret's LGN. (1) How is the sublamination set up in development? LaMantia and Guillery (1982) have found that binocular enucleation in early postnatal ferrets prevents or disrupts even the much clearer cellular lamination which distinguishes lamina $\mathrm{A}$ from $\mathrm{A} 1$, while leaving the $\mathrm{C}$ laminae distinct. This finding suggests that the afferents do have a role in establishing or maintaining lamination of the A laminae. (2) What are the projections of the different sublaminae; are they kept separate up to the level of the cortex? (3) Are the sublaminae themselves stratified with respect to $\mathrm{X}$ and Y input? These questions are currently under study.

Note added in proof. Since this paper went to press, a partial stratification of OFF responses in the LGN of the cat has been reported (Bowling, 1983). Thus, in the tree shrew, rhesus monkey, mink, ferret, and now, cat, OFF activity is always concentrated in the external (nearer the optic tract) half of geniculate laminae. At the same meeting, Norton et al. (1983) reported ON and OFF stratification in layer IV of the striate cortex of tree shrews.

\section{References}

Bowling, D. (1983) Responses to light at different depths in the A layers of the cat's lateral geniculate nucleus. Invest. Ophthalmol. Suppl. 24: 265.

Cleland, B. G., M. W. Dubin, and W. R. Levick (1971) Simultaneous recording of input and output of lateral geniculate neurons. Nature New Biol. 231: 191-192.

Conway, J., P. H. Schiller, and L. Mistler (1980) Functional organization of the tree shrew lateral geniculate nucleus. Soc. Neurosci. Abstr. 6: 583.

Famiglietti, E. V., A. Kaneko, and M. Tachibana (1976) Neuronal architecture of $\mathrm{ON}$ and $\mathrm{OFF}$ pathways to ganglion cells in the carp retina. Science 198: 1267-1269.

Guillery, R. W. (1971) An abnormal retinogeniculate projection in the albino ferret (Mustela furo). Brain Res. 33: 482-485.

Hubel, D. H. (1957) Tungsten microelectrode for recording from single units. Science 125: 549-550.

Hubel, D. H., and T. N. Wiesel (1961) Integrative activity in the cat's lateral geniculate body. J. Physiol. (Lond.) 155: 385398.

Kuffler, S. W. (1953) Discharge patterns and functional organization of mammalian retina. J. Neurophysiol. 16: 37-68.

LaMantia, A. -S., and R. W. Guillery (1982) The effects of binocular enucleation on the development of the dorsal lateral geniculate nucleus (DLGN) of the ferret. Soc. Neurosci. Abstr. 8: 814.

LeVay, S., and S. K. McConnell (1982) ON and OFF layers in the lateral geniculate nucleus of the mink. Nature 300: 350 351.

Linden, D. C., R. W. Guillery, and J. Cucchiaro (1981) The dorsal lateral geniculate nucleus of the normal ferret and its postnatal development. J. Comp. Neurol. 203: 189-211.

Meslaum, M.-M. (1978) Tetramethyl benzidine for horseradish peroxidase neurochemistry: A non-carcinogenic blue reaction-product with superior sensitivity for visualizing neural afferents and efferents. J. Histochem. Cytochem. 26: 106117.

Norton, T. T., R. Kretz, and G. Rager (1983) ON and OFF regions in layer IV of tree shrew striate cortex. Invest. Ophthalmol. Suppl. 24: 265.

Sanderson, K. J. (1974) Lamination of the dorsal lateral geniculate nucleus in carnivores of the weasel (Mustelidae), raccoon (Procyonidae), and fox (Canidae) families. J. Comp. Neurol. 153: 239-266.

Sanderson, K. J., R. W. Guillery, and R. M. Shackelford (1974) Congenitally abnormal visual pathways in mink (Mustela vison) with reduced retinal pigment. .J. Comp. Neurol. 154: 225-248.

Schiller, P. H., and J. G. Malpeli (1978) Functional specificity of lateral geniculate nucleus laminae of the rhesus monkey. J. Neurophysiol. 41: 788-797.

Shatz, C. J., and M. P. Stryker (1978) Ocular dominance in layer IV of the cat's visual cortex and the effects of monocular deprivation. J. Physiol. (Lond.) 281: 267-283.

Zahs, K. R., and M. P. Stryker (1982) The projection of the visual field onto the lateral geniculate nucleus of the ferret. Soc. Neurosci. Abstr. 8: 207. 